\title{
Uso de Redes Generativas Adversariais Aplicadas a Classificação de Sinais de EEG baseado em Imagética Motora*
}

\author{
Lucas H. dos Santos ${ }^{1}$, Henrique V. Giuliani ${ }^{1}$, Patrick O. de Paula ${ }^{1}$, Denis G. Fantinato ${ }^{1}$ \\ ${ }^{1}$ Centro de Matemática, Computação e Cognição \\ Universidade Federal do ABC (UFABC) - Santo André - SP - Brasil \\ \{heck.1, henrique.voni, denis.fantinato\}eufabc.edu.br \\ patrick.oliveiraealuno.ufabc.edu.br
}

\begin{abstract}
The classification of electroencephalography (EEG) signals in BrainComputer Interfaces (BCI) systems is a very challenging task, usually requiring long and exhausting periods of calibration. In this work, the aim is to decreasce the need of collecting many samples for training the classifier through Generative Adversarial Networks (GANs). In addition to that, the classifier shall be developed using cross-subject data, pointing towards a more generic BCI. The results obtained are very promising for the proposal.
\end{abstract}

Resumo. Em sistemas de Interface Cérebro-Computador (BCI, do inglês BrainComputer Interfaces) baseados em imagética motora, a classificação de sinais de eletroencefalografia (EEG) é uma tarefa bastante desafiadora, geralmente exigindo períodos exaustivos de calibração. Neste trabalho buscou-se reduzir a necessidade de muitas amostras coletadas para o treinamento do classificador através das redes adversárias generativas (GANs, do inglês Generative Adversarial Networks). Além disso, o classificador desenvolvido faz o processamento conjunto dos dados dos usuários, visando um sistema BCI mais genérico. Os resultados obtidos com a proposta foram bastante promissores.

\section{Introdução}

A interação humano-máquina por vias não convencionais, como no uso da informação sobre a atividade neural, é interessante não apenas por tornar acessível uma tecnologia assistiva, mas por fomentar novas formas de interfaces, possivelmente mais eficientes ou humanamente mais naturais, para o acionamento de comandos [Nam et al. 2018]. Destacam-se, nesse âmbito, os sistemas de interfaces cérebro-computador (BCI, do inglês Brain-Computer Interfaces), que buscam classificar padrões em registros da atividade cerebral, como a eletroencefalografia (EEG), que é um método não invasivo e de custo relativamente baixo. O resultado da classificação permite o acionamento de comandos em um amplo horizonte de aplicações [Nam et al. 2018].

Recentemente, diversas técnicas de aprendizado profundo (DL, do inglês Deep Learning) têm sido aplicadas ao desenvolvimento de sistemas BCI robustos. A rede convolucional (CNN, do inglês Convolutional Neural Network) [Goodfellow et al. 2016], em particular, vem sendo utilizada como uma promissora estrutura de classificação, inclusive no paradigma de imagética motora, em que o sujeito (usuário do sistema BCI)

\footnotetext{
* O presente trabalho foi realizado com apoio da Coordenação de Aperfeiçoamento de Pessoal de Nível Superior - Brasil (CAPES) - Código de Financiamento 001, e da Fundação de Amparo à Pesquisa do Estado de São Paulo (FAPESP), processo no 2019/17997-4 e 2020/10014-2.
} 
deve imaginar que está realizando o movimento de um ou mais membros/partes do corpo [Nam et al. 2018]. No entanto, as CNNs geralmente dependem de um grande volume de dados para seu adequado treinamento, implicando em grandes períodos de calibração e causando certa exaustão nos usuários desse sistema. Como forma de amenizar esta dependência, neste trabalho é proposta a utilização da rede neural adversária generativa (GAN, do inglês Generative Adversarial Network) [Goodfellow et al. 2016], que pode ser usada para a geração de dados sintéticos. Nesse sentido, a GAN pode ser utilizada para aumentar sinteticamente um conjunto de dados de EEG, sem demandar um período exaustivo para calibração do sistema BCI.

\section{As Interfaces Cérebro-Computador Baseadas em Imagética Motora}

As BCIs baseadas em imagética motora são aquelas que fazem uso da atividade cerebral produzida através do processo voluntário de imaginação de uma tarefa motora. O sujeito deve imaginar, por exemplo, que está realizando a movimentação das mãos, pés ou outros membros do corpo. Essa abordagem baseia-se no fato da atividade neural produzida pela imaginação do movimento ser espaço-temporalmente similar àquela gerada pelo movimento verdadeiramente realizado, mas em menor magnitude [Nam et al. 2018].

A atividade neural, registrada por EEG, pode então ser analisada e classificada em uma das possíveis atividades motoras previamente concebidas. Entretanto, por não haver um sinal ou estímulo de referência (como acontece no paradigma de potenciais evocados), a tarefa de classificação é bastante difícil [Nam et al. 2018]. Neste trabalho é considerado o processamento dos dados de EEG de vários usuários conjuntamente, em que, dado a alta variabilidade observada nos sinais, torna a tarefa ainda mais desafiadora. Para este problema será utilizado como classificador a $\mathrm{CNN}$, que apresenta grande potencial de aprendizado.

\subsection{Redes Neurais Convolucionais}

Uma CNN é um tipo de Rede Neural Artificial (RNA), particularmente adequada para o processamento de tensores (estruturas matriciais $n$ dimensionais) [Goodfellow et al. 2016]. A particularidade das CNNs está no uso da operação de convolução, em uma ou mais camadas da sua estrutura, que envolve a aplicação de um conjunto de filtros, denominados kernels, ao longo de um tensor de entrada. Um kernel é uma estrutura matricial de dimensionalidade consideravelmente reduzida, sendo reutilizado ao longo da entrada, conferindo à convolução maior eficiência computacional e propriedades convenientes para a detecção de informações espaciais, como equivariância para translações [Goodfellow et al. 2016].

De forma a usufruir de um maior potencial das CNNs como classificador em sistemas BCI, os sinais de EEG são transformados em imagens usando o método Gramian Angular Difference Field [Wang and Oates 2015] (GADF). No entanto, para o adequado treinamento das CNNs (ajustes dos pesos que compõem o kernel), uma grande quantidade de dados pode ser necessária, como é o caso em sistemas BCI baseados em imagética motora. Nesse sentido, as GANs serão utilizadas para aumentar sinteticamente o tamanho do conjunto de dados. 


\subsection{Redes Adversárias Generativas}

A GAN é compostas por dois modelos, Gerador e Discriminante, que competem entre si, de forma que, após treinadas, podem gerar um conjunto de dados sintéticos similar ao conjunto real. O Gerador deve gerar amostras que sejam capazes de enganar o Discriminante, impossibilitando-o de distinguir entre genuínas e sintéticas. A arquitetura dos modelos tende a variar, sendo neste trabalho utilizado a DCGAN [Radford et al. 2015] que utiliza CNN em ambos. Na otimização da rede, a função objetivo desempenha um papel relevante para a convergência da GAN. Como meio de resolver alguns problemas provenientes da função original, a Wasserstein GAN (WGAN) foi proposta. Uma das principais melhorias com a WGAN vêm ao adicionar a penalidade nos gradientes do algoritmo de Backpropagation, resultando na WGAN-GP [Gulrajani et al. 2017]. Neste trabalho o objetivo da GAN será o de gerar mais amostras (sintéticas) para o treinamento do classificador.

\section{Resultados}

Para verificar se a GAN, através do incremento do conjunto de dados real com amostras sintéticas, consegue melhorar a classificação dos sinais de EEG foi utilizado o conjunto I da BCI Competition IV [Blankertz et al. 2007] (com taxa de amostragem de $100 \mathrm{~Hz}$ ). Dele foram considerador os quatro usuários reais, com cada um orientado a imaginar a realização de duas ações (dentre três possíveis): mover o braço esquerdo, o direito ou o(s) pé(s). A partir disso extraem-se 800 instâncias/padrões de sinais de EEG, das quais 400 são para o movimento do braço esquerdo e 200 para cada uma das outras. São disponibilizados 64 canais de EEG, dos quais foram selecionados 10 (CFC6, C4, CCP2, CCP4, CCP6, CPz, CP2, CP4, P2 e o P4), que residem em uma região conhecida por exibir maior atividade em ações de imagética motora. Nesses sinais é aplicado o método da média de referência comum [Nam et al. 2018] para reduzir ruídos. Para gerar uma única série temporal é obtida a média de todos os canais. Assim, ao aplicar a transformada GADF é obtida uma única imagem para cada instância.

Para a arquitetura da GAN foi utilizada a DCGAN com a otimização Wasserstein com penalidade nos gradiente. No treinamento usou-se o método Adam para ajuste dos parâmetros com taxa de aprendizagem de $2 \cdot 10^{-4}, \beta_{1}=0$ e $\beta_{2}=0,9$. O treinamento perdurou por 600 épocas com tamanho do lote de 32. Após a conclusão foram geradas amostras sintéticas e comparadas com genuínas visualmente, como disposto na Fig. 1 . Devido a alta variabilidade dos sinais EEG entre usuários distintos é comum ter resultados drasticamente diferentes dentro de uma classe, mas é possível observar que há uma relação entre as regiões mais e menos ativas em amostras de mesma classe tal como entre a genuína e a sintética.
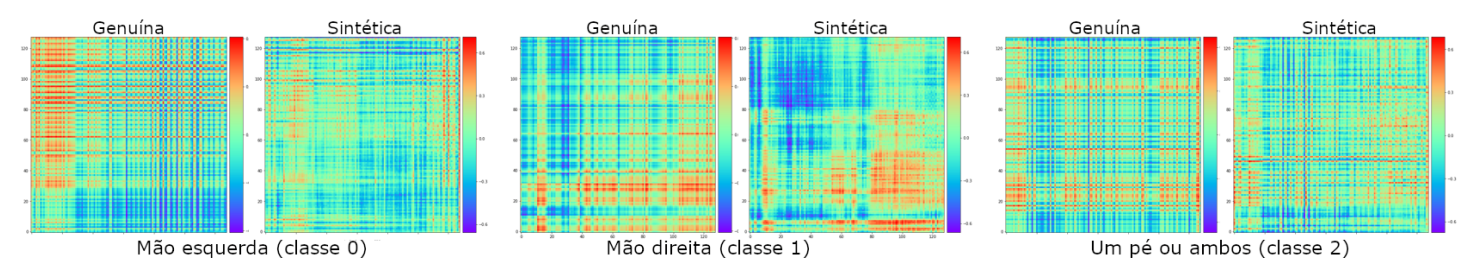

Figura 1. Comparação entre amostras sintéticas geradas pela GAN e amostras genuínas. 
Para classificar as amostras usou-se a arquitetura VGG11 [Simonyan and Zisserman 2014], com diferentes proporções de amostras sintéticas para genuínas. Para o cálculo da acurácia média foi utilizada validação cruzada com 10 pastas. Conforme Fig. 2, é notável a influência positiva ao aumentar o conjunto de treinamento, mesmo sendo de amostras sintéticas. Ao utilizar o conjunto real, o melhor classificador obteve $59,4 \%$ de acurácia, enquanto ao aumentar os dados em $125 \%$, há melhora para $65,6 \%$. Porém, ao incrementar excessivamente em 5 vezes mais amostras (500\%) há uma redução na acurácia, possivelmente porque as amostras genuínas passam a ter menos relevância, causando incertezas no classificador. Como o conjunto real é inicialmente desbalanceado foi introduzida a ideia de balanceá-lo sinteticamente, porém teve impacto negativo. A proporção ótima está no aumento de 3,5 vezes mais amostras (350\%), com um aumento substancial de $13,1 \%$ em relação ao caso original.

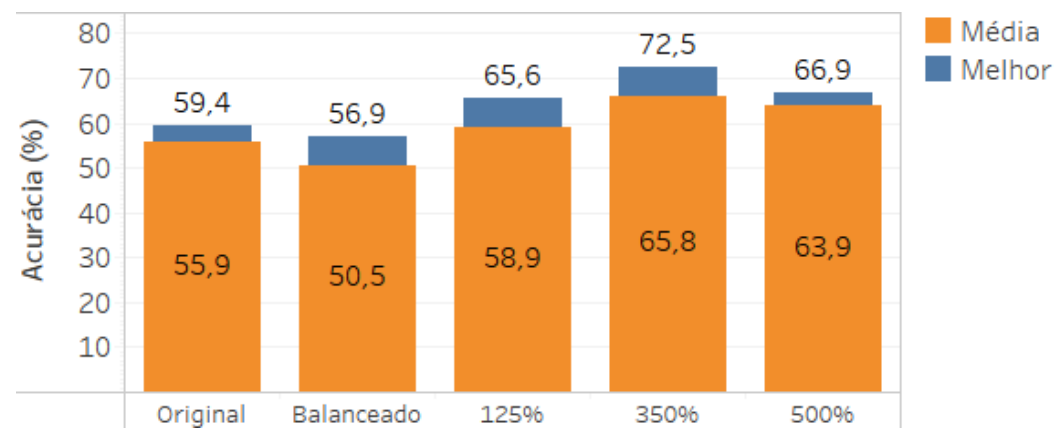

Figura 2. Acurácias do classificador para o conjunto real, balanceado sinteticamente e com diferentes proporções de incremento sintético.

\section{Conclusão}

Neste trabalho foi proposto o uso de GANs para solucionar o problema de poucas amostras disponíveis para treinamento de classificadores de sinais de EEG no paradigma de imagética motora. Foi obtido um ganho substancial na acurácia ao incrementar o conjunto real em até $350 \%$. Como próximos passos, serão analisados outros tipos de mapeamentos dos sinais de EEG para imagens.

\section{Referências}

Blankertz, B., Dornhege, G., Krauledat, M., Müller, K.-R., and Curio, G. (2007). The NonInvasive Berlin Brain-Computer Interface: Fast Acquisition of Effective Performance in Untrained Subjects. NeuroImage, 37(2):539-550.

Goodfellow, I., Bengio, Y., Courville, A., and Bengio, Y. (2016). Deep Learning, volume 1. MIT press Cambridge.

Gulrajani, I., Ahmed, F., Arjovsky, M., Dumoulin, V., and Courville, A. (2017). Improved training of wasserstein gans. arXiv preprint arXiv:1704.00028.

Nam, C. S., Nijholt, A., and Lotte, F. (2018). Brain-Computer Interfaces Handbook: Technological and Theoretical Advances. CRC Press.

Radford, A., Metz, L., and Chintala, S. (2015). Unsupervised Representation Learning with Deep Convolutional Generative Adversarial Networks. arXiv preprint arXiv:1511.06434.

Simonyan, K. and Zisserman, A. (2014). Very Deep Convolutional Networks for Large-Scale Image Recognition. arXiv preprint arXiv:1409.1556.

Wang, Z. and Oates, T. (2015). Imaging Time-Series to Improve Classification and Imputation. arXiv preprint arXiv:1506.00327. 